\title{
Reduced Fertility and Fecundity among Patients with Bipolar I Disorder and Schizophrenia in Egypt
}

\author{
Hader Mansour ${ }^{1,2}$, Kareem Kandil', Joel Wood', Warda Fathi², Mai Elassy², Ibtihal Ibrahim², \\ Hala Salah², Amal Yassin ${ }^{2}$, Hanan Elsayed ${ }^{2}$, Salwa Tobar'2, Hala El-Boraie ${ }^{2}$, Ahmed Eissa², \\ Mohamed Elhadidy ${ }^{2}$, Nahed E. Ibrahim ${ }^{1}$, Wafaa El-Bahaei ${ }^{2}$ and Vishwajit L. Nimgaonkar ${ }^{1,3 凶}$ \\ 'Department of Psychiatry, University of Pittsburgh School of Medicine, Western Psychiatric Institute and Clinic, Pittsburgh, Pennsylvania, USA \\ 2Department of Psychiatry, Mansoura University School of Medicine, Mansoura, Egypt \\ ${ }^{3}$ Department of Human Genetics, Graduate School of Public Health, University of Pittsburgh, Pittsburgh, Pennsylvania, USA
}

Objective To evaluate reproduction among patients with bipolar I disorder (BP1) or schizophrenia (SZ) in Egypt.

Methods BP1 patients $(n=113)$ were compared with community based, demographically balanced controls $(n=124)$ and SZ patients $(\mathrm{n}=79, \mathrm{DSM}-\mathrm{IV})$. All participants were evaluated using structured interviews and corroborative data were obtained from relatives. Standard indices of procreation were included in multivariate analyses that incorporated key demographic variables.

Results Control individuals were significantly more likely to have children than BP1 or SZ patients (controls 46.8\%, BP1 15.9\%, SZ $17.7 \%)$, but the BP1-SZ differences were non-significant. The average number of children for BP1 patients $(0.37 \pm 0.9)$ and SZ patients $(0.38 \pm 0.9)$ was significantly lower than for controls $(1.04 \pm 1.48)$ (BP1 vs controls, $\mathrm{p}<0.001$; SZ vs controls, $\mathrm{p}<0.001)$. The frequency of marriages among BP1 patients was nominally higher than the SZ group, but was significantly lower than controls (BP1: $31.9 \%$ SZ: $27.8 \%$ control: 57.3\%). Even among married individuals, BP1 (but not SZ) patients were childless more often than controls ( $\mathrm{p}=0.001)$. The marital fertility, i.e., the average number of children among patients with conjugal relationships for controls $(1.8 \pm 1.57)$ was significantly higher than BP1 patients $(1.14 \pm 1.31, \mathrm{p}=0.02)$, but not significantly different from SZ patients $(1.36 \pm 1.32, \mathrm{p}=0.2)$.

Conclusion Selected reproductive measures are significantly and substantially reduced among Egyptian BP1 patients. The reproductive indices are similar among BP1 and SZ patients, suggesting a role for general illness related variables. Regardless of the cause/s, the impairment constitutes important, under-investigated disability.

Psychiatry Investig 2011;8:214-220

Key Words Reproduction, Bipolar disorder, Schizophrenia, Fertility, Fecundity.

\section{INTRODUCTION}

Individuals with psychoses may have significant impairment in fertility and fecundity, placing them at a reproductive disadvantage. ${ }^{1-3}$ Patients with schizophrenia (SZ) have been extensively investigated in this regard. ${ }^{4-6}$ Early studies attributed the apparent impairment to prolonged institutionalization, ${ }^{7-10}$ but recent studies have showed similar decreased fertility despite decreased duration of hospital stays. ${ }^{5,6,11-14}$ These results hi-

Received: November 12, 2010 Revised: February 15, 2011

Accepted: February 22, 2011 Available online: August 2, 2011

$\triangle$ Correspondence: Vishwajit L. Nimgaonkar, MD, PhD

Department of Psychiatry, University of Pittsburgh School of Medicine, Western Psychiatric Institute and Clinic, WPIC, Room. 441, 3811 O’Hara Street, Pittsburgh, PA 15213, USA

Tel: +1-412-246-6353 , Fax: +1-412-246-6350, E-mail: nimga+@pitt.edu

(a) This is an Open Access article distributed under the terms of the Creative Commons Attribution Non-Commercial License (http://creativecommons.org/licenses/bync/3.0) which permits unrestricted non-commercial use, distribution, and reproduction in any medium, provided the original work is properly cited. ghlight an important type of disability that has not been emphasized from the perspective of therapeutics.

In contrast to the SZ studies, we are aware of only three systematic studies in the past two decades that have considered procreation among patients with bipolar disorder (BP). Both studies reported on patients from developed countries. In the 1980s, Baron and colleagues evaluated 134 patients with BP and reported that fertility was reduced in particular age groups compared with population US national census data. ${ }^{15}$ A reduction in fertility was also apparent after illness onset, this result was noted more likely among male patients than female patients. A similar finding has been reported by another study conducted in the 1990s, which showed that fertility rates among patients with mood disorder were decreased compared to age matched population norms.,

Several other studies suggest that women with affective disorders have reduced fertility in comparison with appropriate 
comparison groups ${ }^{16-18}$ (reviewed by Williams et al. ${ }^{19}$ ). As many of the studies did not use structured diagnostic criteria, it is uncertain whether they can be generalized to BP individuals using contemporary diagnostic criteria. This question is relevant, as individuals with BP, and particularly those with bipolar I disorder (BP1) may experience increased sexual activity during manic episodes and may experience decreased sexual interest during depressed episodes. The overall impact of such fluctuations on reproduction may therefore be different from patients with depressive disorders.

In the present study, we systematically evaluated a group of patients with BP1 in comparison with community based controls drawn from the same residential areas and socio-economic strata as the cases. The study was conducted in Egypt, where we have previously conducted comprehensive evaluations of BP1 patients. ${ }^{20}$ As the control individuals were screened for absence of BP1, they were not subjected to the impact of illness related variables that may impact procreation, e.g., psychotropic medications. ${ }^{21,22}$ Hence we selected patients with SZ or schizoaffective disorder (SZA) as an additional comparison group. Notably, individuals with SZA have similarities to BP1 patients in some relevant clinical variables, such as types of medication, the course of illness, pre-morbid function and number of hospital admissions. . $^{17,23-25}$

To quantify procreation, we estimated three widely used variables: 1) the number of persons with or without live children; 2) total reproduction rate (TRR), the mean number of children and 3) marital fertility, the mean number of children among married individuals. ${ }^{6,13,26}$ Each of these variables serves as a proxy for a different facet of procreation. The first variable evaluates fertility generally, while TRR estimates fecundity. Both measures are age dependent. They are also contingent on establishing a conjugal relationship, which may be an important hurdle to procreation among individuals with psychiatric illness. ${ }^{27}$ In the rural Egyptian context, where extra marital relationships are uncommon, a conjugal relationship usually follows marriage. Therefore, marital fertility, the third measure indexes fecundity among married individuals.

\section{METHODS}

\section{Recruitment site}

Recruitment was conducted at Mansoura University Hospital (MUH), a Government funded facility that serves as the primary psychiatric care facility for a population of over 7 million individuals from Mansoura and the surrounding villages. This facility is free to all Egyptian citizens. Thus, a variety of urban and rural areas were sampled. As treatment is free, MUH is more likely to treat a more representative set of patients than privately owned fee-for-service facilities. On the oth- er hand, individuals from the lower socio-economic classes are likely to be over-represented in the sample. Mansoura, the capital city of Dakahlia governorate (province) is located 70 miles (112 kms) north of Cairo and 40 miles (64 kms) from the Mediterranean Sea.

\section{Participants}

\section{Cases}

The cases were consenting, unrelated outpatients attending the MUH Psychiatry outpatient clinics who received a clinical diagnosis of BPI, SZ or SZA (DSM IV criteria). Only individuals for whom both parents could provide family history data were included, in order to ensure reliable family history data in both parental lineages. The parents provided family histories, but did not complete diagnostic evaluations.

\section{Controls}

The controls were consenting adults who resided in the same geographic areas as the patients. They were balanced with regard to age and area of residence to the cases. They were recruited over the same period as the cases. Individuals with a history of psychosis or BP1 were excluded from the control group. Available parents were recruited.

\section{Assessment}

Patients and controls were interviewed by trained psychiatrists using the Arabic version of the Schedule for Clinical Assessment in Neuropsychiatry (SCAN), a structured diagnostic interview schedule. ${ }^{28}$ Family history, including parental consanguinity, was obtained using the Arabic version of the Family Interview for Genetic Studies (FIGS). ${ }^{20}$ Occupation alone, rather than a combined index of occupation and education was used as a proxy for socioeconomic status as education is provided freely in Egypt. We used the occupations of the respective heads of households $(\mathrm{HOH})$ rather than individual occupations because in Egypt the occupational status of the $\mathrm{HOH}$ generally reflects the socio-economic status of the entire household. Moreover, many of the female patients listed their occupation as 'housewives', making it difficult to classify their socio-economic status satisfactorily. Consanguinity was defined as marriage between persons who have one common ancestor, no more remote than a great-great grandparent. We have found that self reported consanguinity correspond well with DNA based estimates in this sample. ${ }^{20,29}$

Inter-rater reliability was maintained throughout the study as described in Mansour et al. ${ }^{20}$

\section{Indices of procreation}

Data on procreation were extracted from the SCAN and 
FIGS in a uniform manner from all participants. The term 'marriage' was used to denote legal marriages only. As the ability to have children is restricted by age among women, analyses were conducted only among individuals in the female reproductive age group, defined here as $15-45$ years. Fertility was assessed indirectly as the proportion of patients who reported having one or more live children. Total Reproduction Rate (TRR), defined as the mean number of children per case, is the most popular measure of fecundity. ${ }^{6,26}$ TRR estimates may be biased by the presence of individuals who have never been in conjugal relationships. Therefore, we also estimated the number of individuals who have been married, but are childless. This variable was termed 'marital fertility'. The study was approved by the Mansoura University Ethics Committee and the University of Pittsburgh Institutional Review Board (IRB). All participants provided written informed consent.

\section{Data analysis}

Because the variables of interest were not normally distributed, nonparametric tests were employed where appropriate, and exact 2-tailed significance values are reported. Since procreation is affected by diverse economic and clinical factors, multivariate analysis was conducted in addition to standard univariate comparisons. ${ }^{30}$ All analyses were performed utilizing the Statistical Package for Social Sciences (SPSS, version 17.0 for Windows).

\section{RESULTS}

We initially compared the BP1 patients separately with the control and the SZ groups with regard to demographic variables and indices of procreation. The univariate comparisons between pairs of diagnostic groups were followed by multivar-

Table 1. Demographic variables and reproductive indices

\begin{tabular}{|c|c|c|c|}
\hline Variable* & $\mathrm{BP} 1(\mathrm{n}=113)$ & $\mathrm{SZ}(\mathrm{n}=79)$ & Controls $(\mathrm{n}=124)$ \\
\hline Age & $24.19 \pm 5.47$ & $27.13 \pm 6.55$ & $27.19 \pm 6.7$ \\
\hline Gender (Male/Female) & $55 / 58(48.7 \% / 51.3 \%)$ & $49 / 30(62 \% / 38 \%)$ & $71 / 53(57.3 \% / 42.7 \%)$ \\
\hline Years of education: & $41 / 62 / 6 / 4$ & $14 / 50 / 8 / 5$ & $15 / 59 / 14 / 35$ \\
\hline$(0-8 / 9-12 / 13-15 />15)$ & $(35.4 \% / 54.9 \% / 5.3 \% / 3.5 \%)$ & $(17.7 \% / 63.3 \% / 10.1 \% / 6.3 \%)$ & $(12.1 \% / 47.6 \% / 11.3 \% / 28.2 \%)$ \\
\hline Occupation of head & $4 / 2 / 2 / 5 / 90$ & $6 / 6 / 9 / 6 / 54$ & $17 / 15 / 19 / 12 / 59$ \\
\hline of household ${ }^{\dagger}$ & $(3.5 \% / 1.8 \% / 1.8 \% / 4.4 \% / 79.6 \%)$ & $(7.6 \% / 3.8 \% / 11.4 \% / 7.6 \% / 68.4 \%)$ & $(13.7 \% / 12.1 \% / 15.3 \% / 9.7 \% / 47.6 \%)$ \\
\hline Residence (rural/urban) & $84 / 29(74.3 \% / 25.7 \%)$ & $51 / 27(64.6 \% / 34.2 \%)$ & $92 / 32(74.2 \% / 25.8 \%)$ \\
\hline $\begin{array}{l}\text { Reported parental } \\
\text { consanguinity (yes/no) }\end{array}$ & $41 / 72(36.3 \% / 63.7 \%)$ & $34 / 45(43 \% / 57 \%)$ & 25/99 (20.2\%/79.8\%) \\
\hline Conjugal state & $36 / 76(31.9 \% / 67.3 \%)$ & $22 / 57(27.8 \% / 72.2 \%)$ & $71 / 53(57.3 \% / 42.7 \%)$ \\
\hline \multirow[t]{2}{*}{ (ever married/never married) } & M: $11 / 44$ & M: 5/44 & M: $37 / 34$ \\
\hline & $F: 25 / 32$ & F: $17 / 13$ & F: $34 / 19$ \\
\hline \multirow[t]{3}{*}{ Persons with offspring } & $18(15.9 \%)$ & $14(17.7 \%)$ & $58(46.8 \%)$ \\
\hline & M: 6/49 & M: 3/45 & M: 26/45 \\
\hline & F: $12 / 45$ & F: $11 / 19$ & F: $32 / 21$ \\
\hline \multirow[t]{3}{*}{ Total reproduction rate } & $0.37 \pm 0.91$ & $0.38 \pm 0.929$ & $1.04 \pm 1.48$ \\
\hline & M: $0.24 \pm 0.693$ & M: $0.08 \pm 0.347$ & M: $0.79 \pm 1.463$ \\
\hline & F: $0.49 \pm 1.07$ & F: $0.87 \pm 1.306$ & F: $1.38 \pm 1.457$ \\
\hline \multirow[t]{3}{*}{ Marital fertility } & $1.14 \pm 1.31$ & $1.36 \pm 1.32$ & $1.8 \pm 1.57$ \\
\hline & M: $1.18 \pm 1.168$ & M: $0.80 \pm 0.837$ & M: $1.51 \pm 1.742$ \\
\hline & F: $1.12 \pm 1.394$ & $F: 1.53 \pm 1.419$ & F: $2.12 \pm 1.320$ \\
\hline
\end{tabular}

Age and Total Reproduction Rate are reported as mean \pm standard deviation. Marital fertility: mean number of children among individuals who have been married or been in conjugal relationships. * some variables could not be obtained for a minority of participants, ${ }^{\dagger}$ occupations were considered under the following categories: Managerial and professional, Technical and sales, Administrative, Service occupation, others. BP1: bipolar I disorder, SZ: schizophrenia, SD: standard deviation, M: men, F: women

Significant group-wise comparisons

Education: BP1 vs control: $\chi^{2}=22.092$, $p=0.00006$, d.f. 3; BP1 vs SZ: $\chi^{2}=1.18, p=0.7$, d.f. 3; SZ vs control: $\chi^{2}=15.135$, $p=0.001$, d.f. 3

Occupation of head of household: BP1 vs control: $\chi^{2}=39.76, p \leq 0.001$, d.f. 3 ; BP1 vs SZ: $\chi^{2}=13.5, p=0.009$, d.f. 3; SZ vs control: $\chi^{2}=6.9$, $p=0.1$, d.f. 3 Parental consanguinity: BP1 vs control: $\chi^{2}=7.64, p=0.005$, d.f. 1 ; BP1 vs $S Z: \chi^{2}=0.89, p=0.3$, d.f. 1 ; $S Z$ vs control: $\chi^{2}=12.25, p=0.0004$, d.f. 1 Conjugal state BP1 vs control: $\chi^{2}=14.97$, $\mathrm{p}=0.0001$, d.f. 1 ; BP1 vs SZ: $\chi^{2}=0.4, \mathrm{p}=0.5$, d.f. 1 ; SZ vs control: $\chi^{2}=16.81$, $\mathrm{p}=0.00004$, d.f. 1 Persons with offspring: BP1 vs control: $\chi^{2}=25.40$, $p \leq 0.001$, d.f. 1 ; BP1 vs $S Z: \chi^{2}=0.011$, $p=0.7$, d.f. 1 ; $S Z$ vs control: $\chi^{2}=17.34$, $p \leq 0.001$, d.f. 1 Total Reproduction Rate BP1 vs control $z=-4.75, p \leq 0.001$; BP1 vs $S Z: Z=-0.284, p=0.79$; $S Z$ vs control $z=-3.98, p \leq 0.001$ (Mann Whitney $U$ test) Married persons with offspring: BP1 vs control: $\chi^{2}=10.44$, $p=001$, d.f. 1 ; $B P 1$ vs SZ: $\chi^{2}=1$, $p=0.3$, d.f. 1 ; SZ vs control: $\chi^{2}=2.57, p=0.1$, d.f. 1 
iate analyses that also included relevant demographic and clinical variables.

\section{Group-wise comparisons: demographic and clinical variables}

The participants included 113 patients diagnosed with BP1, 76 patients with SZ, 3 patients with SZA and 124 controls (Table 1). Due to the relatively small number, the SZA patients were analyzed together with the SZ patients. All analyses were restricted to individuals between the ages of 15-45 years. The BP1 patients did not differ significantly from the SZ or the control group with regard to age, gender, or area of residence (rural/urban). However, the BP1 and SZ patients had significantly lower levels of education compared with the control group. As reported before, the rates of parental consanguinity were also significantly higher in the BP1 and the SZ groups compared with the control individuals. ${ }^{20,29}$ None of these variables were significantly different between the BP1 and SZ groups. The occupation of the heads of households in the BP1 group was significantly lower than in the SZ and control groups (Table 1).

\section{Group-wise comparisons: variables related to procreation}

While $57.3 \%$ of the control group reported that they had ever been in conjugal relationships, $31.9 \%$ of the BP1 group, and $27.8 \%$ of the SZ patients reported that they had been in conjugal relationships.

Substantially fewer patients with BP1 (15.9\%) reported having offspring in comparison with the control group (46.8\%, $\left.\chi^{2}=25.4, \mathrm{p} \leq 0.001\right)$. The rates among the SZ patients were similar to the BP1 group (17.7\%) and were substantially lower than the control individuals $\left(\chi^{2}=17.34, \mathrm{p} \leq 0.001\right)$. The TRR, defined as the number of children per patient, among BP1 $(0.37 \pm 0.9$ and SZ patients $(0.38 \pm 0.92)$ was significantly lower than the control (1.4 \pm 1.48$)$ group ( $\mathrm{p} \leq 0.001$ and $\mathrm{p}<0.001$ respectively). The lower TRR in the BP1 and SZ groups may be due to disproportionately larger number of individuals who never established conjugal relationships, as well as infertile individuals. Therefore, we also evaluated marital fertility, defined here as the mean number of children among individuals who had been married or had been in conjugal relationships. Married BP1 patients are more likely to be childless than married controls (marital fertility: BP1: 1.14 $\pm 1.31, \mathrm{SZ}: 1.36 \pm 1.32$, controls: $1.8 \pm 1.57 ; \mathrm{p}=0.02$ ). The marital fertility was significantly lower among the BP1 patients compared with controls, but there were no significant differences between the BP1 and the SZ patients or between the SZ patients and the controls.

These patterns were observed among male as well as female patients. The case-control differences were more prominent among male patients, particularly among the SZ patients (Table 1). Prescription or non-prescription of antipsychotic medication was not significantly associated with the number of offspring (data not shown).

\section{Multivariate analyses}

The concurrent association of selected demographic and clinical variables with fertility and fecundity were next evaluated. Fertility was examined among the participants using logistic regression analysis. Presence or absence of offspring was considered as the outcome variable. Logistic regression analyses suggested that being a patient with BP1 or SZ significantly predicts presence/absence of live offspring after taking into ac-

Table 2. Logistic regression using presence of children as the outcome among BP1 and control groups

\begin{tabular}{lcccccccc}
\hline \multirow{2}{*}{ Covariate } & $\beta$ coefficient & Standard error & Wald & df & p value & Exp (B) & \multicolumn{2}{c}{ 95\% CI for exp (B) } \\
\hline Sex & -0.988 & 0.496 & 3.960 & 1 & 0.047 & 0.372 & 0.141 & 0.985 \\
Age & 0.075 & 0.043 & 3.144 & 1 & 0.076 & 1.078 & 0.992 & 1.172 \\
Consanguinity & -0.443 & 0.555 & 0.637 & 1 & 0.425 & 0.642 & 0.216 & 1.906 \\
Marital Status & -5.217 & 1.085 & 23.141 & 1 & $<0.001$ & 0.005 & 0.001 & 0.045 \\
Residence & 0.115 & 0.584 & 0.039 & 1 & 0.843 & 1.122 & 0.357 & 3.527 \\
HOH & & & 3.318 & 4 & 0.506 & & & \\
HOH (1) & -0.173 & 0.888 & 0.038 & 1 & 0.845 & 0.841 & 0.148 \\
HOH (2) & 1.213 & 1.015 & 1.428 & 1 & 0.232 & 3.364 & 0.460 & 24.600 \\
HOH (3) & -0.679 & 0.850 & 0.639 & 1 & 0.424 & 0.507 & 0.096 \\
HOH (4) & 0.969 & 1.043 & 0.862 & 1 & 0.353 & 2.635 & 0.341 \\
Diagnostic status & 1.559 & 0.533 & 8.539 & 1 & 0.003 & 4.752 & 2.681 \\
Constant & -1.645 & 1.494 & 1.212 & 1 & 0.271 & 0.193 & 20.360 \\
\hline
\end{tabular}

Variable(s) entered: sex, age, consanguinity, marital status, residence (urban/rural), and occupation of the head of household (HOH) which was considered under the following categories (Managerial and professional, Technical and sales, Administrative, Service occupation, others). BP1: bipolar I disorder, CI: confidence interval 
Table 3. Logistic regression using presence of children as the outcome among SZ and control groups

\begin{tabular}{lcccccccc}
\hline \multirow{2}{*}{ Covariate } & $\beta$ coefficient & Standard error & Wald & df & p value & Exp (B) & \multicolumn{2}{c}{ 95\% CI for exp (B) } \\
\hline Sex & -1.661 & 0.610 & 7.418 & 1 & 0.006 & 0.190 & 0.057 & 0.628 \\
Age & 0.057 & 0.044 & 1.688 & 1 & 0.194 & 1.059 & 0.971 & 1.154 \\
Consanguinity & -0.739 & 0.673 & 1.204 & 1 & 0.272 & 0.478 & 0.128 & 1.787 \\
Marital Status & -5.912 & 1.259 & 22.059 & 1 & $<0.001$ & 0.003 & 0.000 & 0.032 \\
Residence & -0.266 & 0.686 & 0.151 & 1 & 0.698 & 0.766 & 0.200 & 2.940 \\
HOH & & & 5.884 & 4 & 0.208 & & & \\
HOH (1) & 0.468 & 0.902 & 0.270 & 1 & 0.604 & 1.597 & 0.273 \\
HOH (2) & 2.928 & 1.552 & 3.557 & 1 & 0.059 & 18.686 & 0.891 & 391.674 \\
HOH (3) & -0.743 & 0.802 & 0.860 & 1 & 0.354 & 0.476 & 0.099 \\
HOH (4) & 1.802 & 1.494 & 1.456 & 1 & 0.228 & 6.064 & 0.324 & 113.339 \\
Diagnostic status & 1.676 & 0.696 & 5.797 & 1 & 0.016 & 5.345 & 1.366 & 20.919 \\
Constant & -0.449 & 1.608 & 0.078 & 1 & 0.780 & 0.638 &
\end{tabular}

Variable(s) entered: sex, age, consanguinity, marital status, residence (urban/rural), and occupation of the head of household (HOH) which was considered under the following categories (Managerial and professional, Technical and sales, Administrative, Service occupation, others). SZ: schizophrenia, CI: confidence interval

count age, gender, residence (urban/rural), marital status and consanguinity (BP1: $\mathrm{p}=0.003, \mathrm{OR}=4.75,95 \% \mathrm{CI}=1.67-13.51$; SZ: $\mathrm{p}=0.01, \mathrm{OR}=5.34,95 \% \mathrm{CI}=1.36-20.9)$ (Table 2 and 3).

Likewise, Poisson regression analyses using number of children as the outcome showed that being a patient with BP1 or SZ significantly predicts the TRR taking into account the same demographic variables ( $\mathrm{p}=0.012$ for BP1 and 0.02 for SZ) (Data not shown).

\section{DISCUSSION}

To our knowledge, this is the first study to systematically estimate procreation among BP1 and SZ patients in Egypt, using modern diagnostic criteria. Relatively accurate indices of reproduction may be obtained from this population, as procreation typically occurs in the course of conventional marriages. Our analyses suggest that all three indices of procreation are significantly and substantially impaired among BP1 patients in comparison with community based controls, even after key demographic variables were included in multivariate analyses. Our results are counterintuitive, because BP1 patients in manic states typically have increased libido and thus would be expected to have increased procreation. The predicted increase may be counterbalanced by prolonged depressive states, which are known to be associated with impaired fertility. ${ }^{16}$ The BP1 patients also have reduced frequency of marital relationships, possibly related to stigma. Our results thus indicate an important type of disability among the BP1 patients.

The reduced procreation observed among the BP1 patients is similar to the SZ patients, a group that has been widely reported to have impaired procreation. Thus, our observations may not indicate a deficit specific to BP1. Rather, they may reflect the impact of more general illness related variables. ${ }^{25,31}$ Antipsychotic drugs, the medications likely to be implicated did not appear to impact the number of offspring in our study. Other possible illness related factors include duration and state of illness (remission/relapse), and co-morbid substance abuse. Reproduction is a complex function, with a significant impact of variables such as age, general effects of illness on sexual function, age at marriage, a desire for having children, attempts to conceive, use of birth control measures, or presence of organic factors leading to infertility. ${ }^{19}$ As systematic information was not available, the relative contributions of these factors could not be addressed individually in the present study. A number of other explanatory models have been proposed for the reproductive impairment also observed in depressive disorder, such as variation in hormonal levels. ${ }^{19,32-34}$ Such mechanisms may also explain our observations. Others have suggested that the reductions reflect evolutionary forces, but this question is complex. ${ }^{35}$ Regardless of the mechanisms, our results merit replicate studies. It would also be important to assess BP1 patients from other ethnic groups in order to evaluate cultural variations.

We also observed significant gender related differences among the patients. In the BP1 group, men were less likely to have children and to have lower TRR values compared with female BP1 patients. These gender differences are consistent with generally observed differences in many populations and were also observed among our community based control sample. Consistent with earlier reports among US and Indian samples, the gender related trends were accentuated in the SZ group, with sharply reduced TRR as well as higher rates of childlessness among male SZ patients. ${ }^{6,22,30,36}$ In contrast to the male SZ 
patients, the marital fertility among men with BP1 patients was similar to women with BP1. Thus, the lower TRR among male BP1 patients overall may be related to difficulties in establishing conjugal relationships. Indeed, fewer men with $\mathrm{BP} 1$ were married, in comparison with female BP1 patients.

All the measures of procreation used in this study were based on self-report. To reduce chances of under-reporting, we obtained corroborative information from the parents of the participants. However, detailed information could not be obtained about the diagnostic status of the patients' spouses. Psychiatric illness in the spouses or other relatives may also lead to voluntary cessation of reproduction in some instances..$^{25,37}$ Though data about childbirth outside conjugal relationships were not collected during our study, such events are relatively infrequent. Nevertheless, they could be a source of error in our estimates.

In conclusion, measures of fertility and fecundity among Egyptian BP1 patients are significantly lower than control individuals and are comparable to estimates for SZA/SZ patients. The differences are unlikely to be due to common demographic variables. Further studies controlling for other important variables such as illness severity, birth control use and hormonal levels are warranted. These results may be relevant for genetic models of causation, which typically do not incorporate the impact of reduced procreation.

\section{Acknowledgments}

We thank participants in our studies, as well as faculty members at the Department of Psychiatry, MUH. Funded in part by grants from the Fogarty International Center, National Institute of Health (FIC, NIH) (MH 63420, TW006949 and TW007997 to VLN and D43 TW008302 to VLN / MH).

\section{REFERENCES}

1. Odegård O. Marriage and mental disease; a study in social psychopathology. J Ment Sci 1946;92:35-59.

2. Odegård $\mathrm{O}$. Marriage rate and fertility in psychotic patients before hospital admission and after discharge. Int J Soc Psychiatry 1960;6:25-33.

3. Laursen TM, Munk-Olsen T. Reproductive patterns in psychotic patients. Schizophr Res 2010;121:234-240.

4. Stevens B. Marriage and Fertility of Women Suffering from Schizophrenia or Affective Disorders. London: Oxford University Press; 1969.

5. Ritsner M, Sherina O, Ginath Y. Genetic epidemiological study of schizophrenia: reproduction behaviour. Acta Psychiatr Scand 1992;85: 423-429.

6. Nimgaonkar VL. Reduced fertility in schizophrenia: here to stay? Acta Psychiatr Scand 1998;98:348-353.

7. Odegård $\mathrm{O}$. New data on marriage and mental disease: the incidence of psychoses in the widowed and the divorces. J Ment Sci 1953;99:778-785.

8. Odegård O. Fertility of psychiatric first admissions in Norway 19361975. Acta Psychiatr Scand 1980;62:212-220.

9. Haverkamp F, Propping P, Hilger T. Is there an increase of reproductive rates in schizophrenics? I. Critical review of the literature. Arch Psychiatr Nervenkr 1982;232:439-450.

10. Saugstad LF. Social class, marriage, and fertility in schizophrenia. Schizophr Bull 1989;15:9-43.

11. Fañanás L, Bertranpetit J. Reproductive rates in families of schizo- phrenic patients in a case-control study. Acta Psychiatr Scand 1995;91: 202-204.

12. Goldstein JM, Faraone SV, Chen WJ, Tolomiczencko GS, Tsuang MT. Sex differences in the familial transmission of schizophrenia. Br J Psychiatry 1990;156:819-826.

13. Nanko S, Moridaira J. Reproductive rates in schizophrenic outpatients. Acta Psychiatr Scand 1993;87:400-404.

14. Bassett AS, Bury A, Hodgkinson KA, Honer WG. Reproductive fitness in familial schizophrenia. Schizophr Res 1996;21:151-160.

15. Baron M, Risch N, Mendlewicz J. Differential fertility in bipolar affective illness. J Affect Disord 1982;4:103-112.

16. Jönsson SA. Marriage rate and fertility in cycloid psychosis: comparison with affective disorder, schizophrenia and the general population. Eur Arch Psychiatry Clin Neurosci 1991;241:119-125.

17. Calzeroni A, Conte G, Pennati A, Vita A, Sacchetti E. Celibacy and fertility rates in patients with major affective disorders: the relevance of delusional symptoms and suicidal behaviour. Acta Psychiatr Scand 1990; 82:309-310.

18. Harlow BL, Wise LA, Otto MW, Soares CN, Cohen LS. Depression and its influence on reproductive endocrine and menstrual cycle markers associated with perimenopause: the Harvard Study of Moods and Cycles. Arch Gen Psychiatry 2003;60:29-36.

19. Williams KE, Marsh WK, Rasgon NL. Mood disorders and fertility in women: a critical review of the literature and implications for future research. Hum Reprod Update 2007;13:607-616.

20. Mansour H, Klei L, Wood J, Talkowski M, Chowdari K, Fathi W, et al. Consanguinity associated with increased risk for bipolar I disorder in Egypt. Am J Med Genet B Neuropsychiatr Genet 2009;150B:879-885.

21. Petty RG. Prolactin and antipsychotic medications: mechanism of action. Schizophr Res 1999;35(Suppl):S67-S73.

22. Howard LM, Kumar C, Leese M, Thornicroft G. The general fertility rate in women with psychotic disorders. Am J Psychiatry 2002;159: 991-997.

23. Vogel HP. Fertility and sibship size in a psychiatric patient population. A comparison with national census data. Acta Psychiatr Scand 1979; 60:483-503.

24. Hutchinson G, Bhugra D, Mallett R, Burnett R, Corridan B, Leff J. Fertility and marital rates in first-onset schizophrenia. Soc Psychiatry Psychiatr Epidemiol 1999;34:617-621.

25. Hendrick V, Gitlin M, Altshuler L, Korenman S. Antidepressant medications, mood and male fertility. Psychoneuroendocrinology 2000;25: 37-51.

26. Bhatia T, Franzos MA, Wood JA, Nimgaonkar VL, Deshpande SN. Gender and procreation among patients with schizophrenia. Schizophr Res 2004;68:387-394.

27. Erlenmeyer-Kimling L, Nicol S, Rainer J, Deming W. Changes in fertility rates of schizophrenic patients in New York state. Am J Psychiatry 1969;125:916-927.

28. Wing JK, Sarotorius N, Ustun TB. Diagnosis and Clinical Measurement in Psychiatry: A Reference Manual for Scan. Cambridge: Cambridge University Press; 1998.

29. Mansour H, Fathi W, Lambertus K, Wood J, Chowdari KV, Watson A, et al. Consanguinity and increased risk for schizophrenia in Egypt. Presented at the XVII World Congress on Psychiatric Genetics. San Diego, CA; 2009.

30. Nimgaonkar VL, Ward SE, Agarde H, Weston N, Ganguli R. Fertility in schizophrenia: results from a contemporary US cohort. Acta Psychiatr Scand 1997;95:364-369.

31. Currier GW, Simpson GM. Antipsychotic medications and fertility. Psychiatr Serv 1998;49:175-176.

32. Downey J, Yingling S, McKinney M, Husami N, Jewelewicz R, Maidman J. Mood disorders, psychiatric symptoms, and distress in women presenting for infertility evaluation. Fertil Steril 1989;52:425-432.

33. Fassino S, Pierò A, Boggio S, Piccioni V, Garzaro L. Anxiety, depression and anger suppression in infertile couples: a controlled study. Hum Re- 
prod 2002;17:2986-2994.

34. Meller W, Burns LH, Crow S, Grambsch P. Major depression in unexplained infertility. J Psychosom Obstet Gynaecol 2002;23:27-30.

35. Adriaens PR. Debunking evolutionary psychiatry's schizophrenia paradox. Med Hypotheses 2008;70:1215-1222.

36. Bhatia T, Franzos MA, Wood JA, Nimgaonkar VL, Deshpande SN.
Gender and procreation among patients with schizophrenia. Schizophr Res 2004;68:387-394.

37. Waddington JL, Youssef HA. Familial-genetic and reproductive epidemiology of schizophrenia in rural Ireland: age at onset, familial morbid risk and parental fertility. Acta Psychiatr Scand 1996;93:62-68. 